\title{
Monolithic Integration of InGaAs p-i-n Photodetector with Fully Ion-Implanted InP JFET Amplifier
}

SUNG J. KIM, MEMBer, IEEe, G. GUTH, G. P. VELlA-COLEIRO, MEMBER, IEeE, CHARLES W. SEABURY, MEMBER, IEEe, W. A. SPONSLER, AND B. J. RHOADES

\begin{abstract}
A monolithically integrated p-i-n FET amplifier has been fabricated using ion-implanted indium phosphide (InP) JFET's. The vertically integrated material structure consists of a vapor phase epitaxy (VPE) grown InGaAs photoabsorption layer and a metal organic chemical vapor deposition (MOCVD) grown Fe-doped semi-insulating layer. A Zn diffusion was performed to complete the p-i-n photodiode. High-performance fully implanted InP JFET's were used to form the integrated amplifier with a symmetrical design to remove the dc offset. With a receiver sensitivity of $-36.4 \mathrm{dBm}$ measured at $200-\mathrm{Mbit} / \mathrm{s} \mathrm{NRZ}$ for $10{ }^{~} \mathrm{BER}$, it is easily the most sensitive monolithic p-i-n FET preamp yet reported in this frequency range. The $p$-i-n amplifier has a dynamic range of $15 \mathrm{~dB}$.
\end{abstract}

\section{INTRODUCTION}

$\mathrm{T}$ HE OPTICAL communication systems developed in this decade use both short- $(0.8-0.9 \mu \mathrm{m})$ and long- $(1.3-1.6$ $\mu \mathrm{m})$ wavelength regions, but due to the lower attenuation transmission windows in silica fibers in the long-wavelength region, the GaInAsP alloy materials grown lattice matched to indium phosphide (InP) have drawn much attention. The InPbased optoelectronics integrated circuits (OEIC's), therefore, have become an important component for communication systems to be built in the future. In order to build such integrated circuits, one requires a good electronics technology. Unlike Si and GaAs, however, InP does not have a wellestablished FET technology. Due to gate-semiconductor interfacial problems, the metal-insulator-semiconductor (MIS) and metal-semiconductor (MES) FET's do not appear to be practical in InP. InP JFET's have demonstrated good performance and may be the device of choice until the problems with other options can be solved.

Several groups have reported long-wavelength integrated $p$-i-n FET photodetector devices using InP/InGaAs FET's operating at various bit rates [1]-[5], but the sensitivities were relatively low. In [6], an alternative approach has been taken to fabricate MESFET's on a GaAs layer grown lattice mismatched to an InP substrate. Among the reported $p-i-n$ FET devices, only [5] and [6] employ integration of a

Manuscript received May 5, 1988; revised June 24. 1988

S. J. Kinl, G. Guth, G. P. Vella-Colciro, and C. W. Seabury are with AT\&T Bell Laboratories. Murray Hill, NJ 07974.

W. A. Sponsler and B. J. Rhoades are with AT\&T Microelectronics, Reading, PA 19604.

IEEE Log Number 8823106 complete amplifier, including a level-shifting buffer stage. We report in this paper the fabrication and performance of an integrated p-i-n FET amplifier with high sensitivity. The amplifier is made using high-performance fully ion-implanted InP JFET's and, therefore, only materials grown lattice matched to an InP substrate are used.

\section{Device Structure and Fabrication}

Fig. 1 shows schematically the structure of the integrated $\mathrm{p}-\mathrm{i}-\mathrm{n}$ JFET device. The material was grown on an $\mathrm{n}^{+}-\mathrm{InP}$ substrate in two steps. The first growth was done by chloride vapor phase epitaxy (VPE) [7]. Following the growth of an undoped InP buffer, a 3.5- $\mu$ m-thick $\operatorname{In}_{0.53} \mathrm{Ga}_{0.47}$ As layer with an electron concentration less than $1 \times 10^{15} \mathrm{~cm}^{-3}$ was grown and capped by another layer of undoped InP. The second growth was done by atmospheric pressure metal organic chemical vapor deposition (MOCVD) [8], [9] and resulted in a 3- $\mu$ m-thick Fe doped semi-insulating InP layer with a typical resistivity higher than $10^{7} \Omega \cdot \mathrm{cm}$.

The wafer with grown epilayers was then subjected to a series of ion implantations to form the JFET. The details of the ion implantation and activation are similar to those reported earlier [10]. The channel was formed by implanting ${ }^{29} \mathrm{Si}$, and it was followed by the source-drain implant to form highly doped regions. Subsequently, As/Be coimplant was used to form the $\mathrm{p}^{+}$gate region. All implants were selective, non-selfaligned using $\mathrm{SiN}_{x}$ and photoresist as an implantation mask. The implanted wafer was then activated in a rapid thermal annealer (RTA) using a dielectric capping layer.

Following deposition of a $\mathrm{SiN}_{x}$ passivation layer, the $\mathrm{Zn}$ diffusion to form the $\mathrm{p}$-i-n diode was performed using an evaporated film of zinc phosphide patterned by a lift-off. Diffusion was performed in an RTA, and the resulting p-n junction was located near the InP-InGaAs interface. Further details of the p-i-n fabrication will be reported elsewhere. The rest of the device processing consists of three metallization steps: for the gates, sources and drains, and interconnects.

\section{Device Performance}

Previously, we reported [10], [11] on the fabrication of fully ion-implanted InP JFET's on semi-insulating (SI) substrates. We have fabricated JFET's with similar performance 


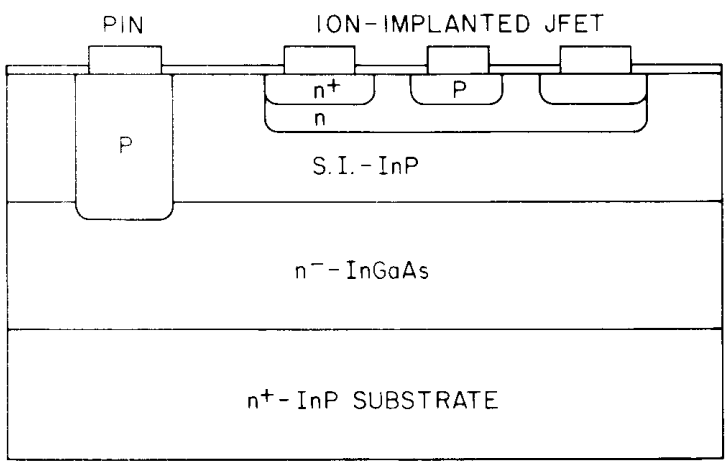

Fig. 1. Cross-sectional view of the integrated $p$-i-n JFET device.
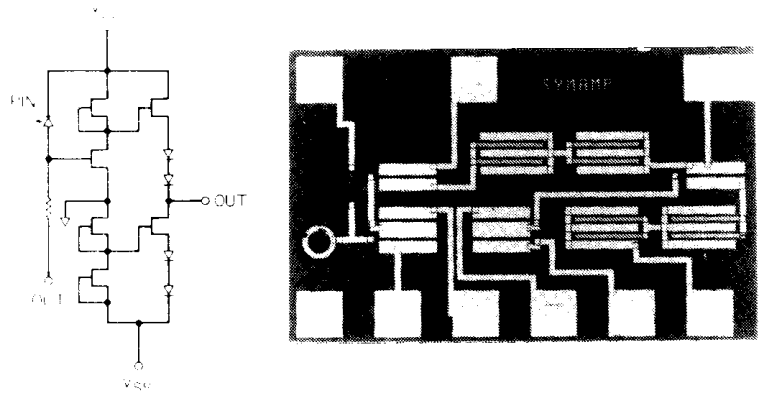

Fig. 2. p. I JFET symmetrical amplifier circuit diagram and microphotograph of a fabricated chip.

on the MOCVD-grown SI layer. From the JFET's used in this work, a transconductance $g_{m}$ of $100 \mathrm{mS} / \mathrm{mm}$ was measured at $V_{g s}=0$. With $C_{g s}$ of $1.8 \mathrm{pF} / \mathrm{mm}$, a 3 -dB cutoff frequency $g_{m} /$ $\left(2 \pi C_{k s}\right)$ of $9 \mathrm{GHz}$ is calculated.

Fig. 2 shows the circuit diagram of the integrated $p-i-n$ JFET amplifier and the microphotograph of a fabricated chip. The $\mathrm{p}$-i-n's are fabricated into a 75- $\mu \mathrm{m}$-diameter circle. Within the circle, the p-contact metal is defined as a $10-\mu \mathrm{m}$ wide ring. The $p-i-n$ has a dark current of $10 \mathrm{nA}$ and a capacitance of $0.25 \mathrm{pF}$ at $-5 \mathrm{~V}$. A $30-\mathrm{k} \Omega$ resistor was also fabricated using the channel implant to serve as a feedback resistor for the amplifier.

The amplifier consists of a symmetrical design which, as described in [11], results in negligible dc output offset. All FET's are $100 \mu \mathrm{m}$ width, and the level-shifting diodes are implemented with p-n junctions $10 \times 200 \mu \mathrm{m}^{2}$ in area. At $V_{\text {in }}$ $=0$, an open-loop voltage gain of 5.5 was measured from the amplifier.

The fabricated p-i-n amplifier circuit has been mounted on a ceramic carrier for optical sensitivity measurements. Bondwire connections were made from the chip to the ceramic carrier for power supply and ground connections and also to form the transimpedance configuration. Only two powersupply voltages ( $\left.V_{d d}=-V_{s s}=5 \mathrm{~V}\right)$ were used.

A bandwidth of $40 \mathrm{MHz}$ was measured from the p-i-n amplifier in transimpedance configuration. The bandwidth was limited by the $R C$ delay at the amplifier input where $R$ is the effective resistance (feedback resistance/(open-loop gain

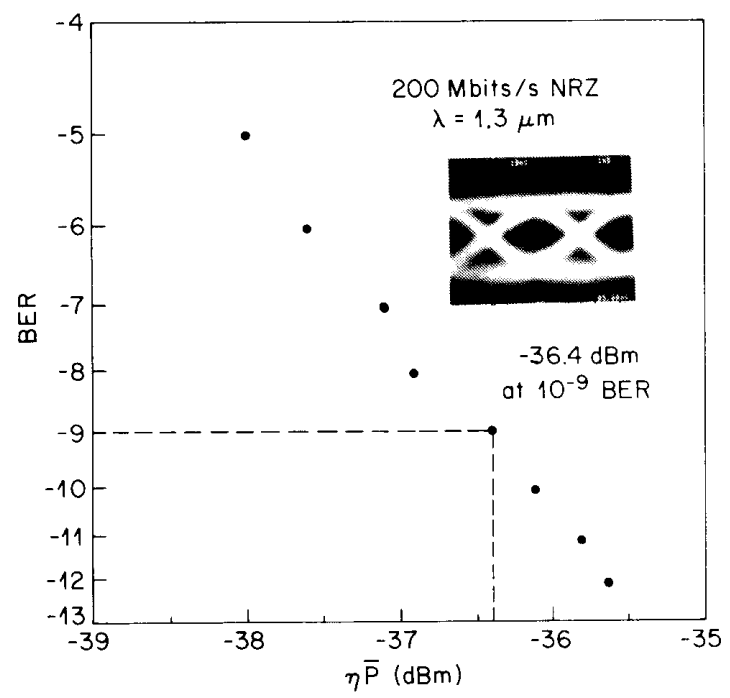

Fig. 3. Detector sensitivities measured for several BER's. The insert shows an eye diagram taken at $200-\mathrm{Mbit} / \mathrm{s}$ NRZ for $10^{-9} \mathrm{BER}$.

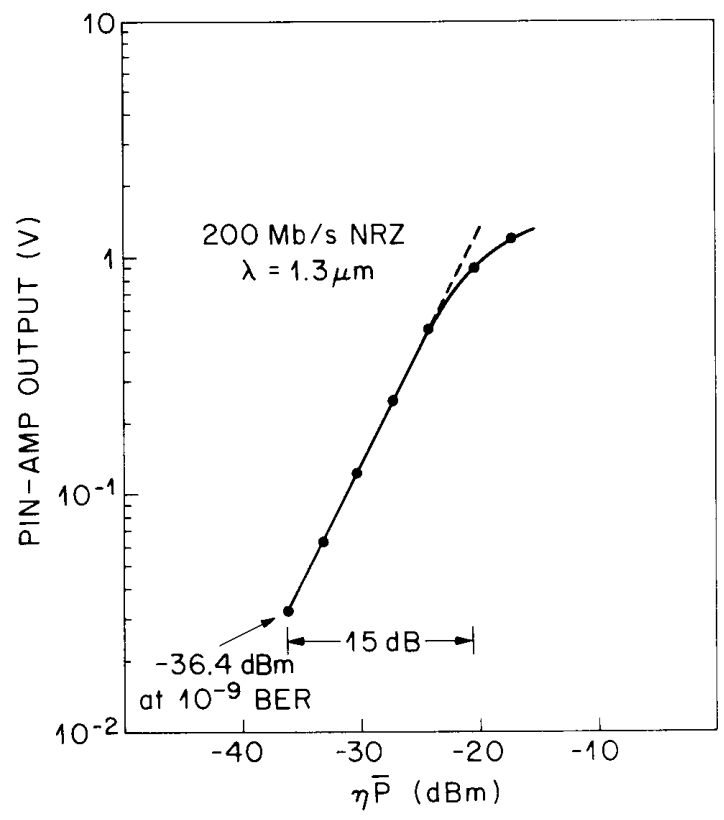

Fig. 4. Peak-to-peak amplifier output voltage versus detected optical power A dynamic range of $15 \mathrm{~dB}$ is measured.

$+1)$ ) and $C$ is the total capacitance (p-i-n, FET, and stray capacitance). The output was equalized to obtain a $100-\mathrm{MHz}$ bandwidth.

The sensitivity measurement was performed at 200-Mbit/s NRZ format. Detected power was measured for several biterror rates (BER's) (Fig. 3). For $10^{-9}$ BER, the sensitivity was $-36.4 \mathrm{dBm}$. We believe that this is the best sensitivity reported to date from a $\mathrm{p}-\mathrm{i}-\mathrm{n}$ FET device designed for 1.3$1.55-\mu \mathrm{m}$ wavelength at this bit rate. The insert in Fig. 3 shows an eye diagram taken at $200 \mathrm{Mbit} / \mathrm{s}$ for $10^{-9} \mathrm{BER}$.

Fig. 4 shows the peak-to-peak amplifier output voltage as a 
function of detected optical power. For every 3-dB increment of the optical power, the amplifier output doubles until it saturates. From the minimum detected power of $-36.4 \mathrm{dBm}$ (at $10^{-9} \mathrm{BER}$ ) to the saturated knee point, a dynamic range of $15 \mathrm{~dB}$ is measured.

\section{Conclusion}

An InGaAs p-i-n has been monolithically integrated with a full amplifier circuit using InP JFET's to achieve high sensitivity. The fully implanted FET's were fabricated on an MOCVD-grown semi-insulating InP layer together with a diffused $\mathrm{p}-\mathrm{i}-\mathrm{n}$. A receiver sensitivity of $-36.4 \mathrm{dBm}$ was measured at $200-\mathrm{Mbit} / \mathrm{s} \mathrm{NRZ}$ for $10^{-9} \mathrm{BER}$. This sensitivity can be improved by reducing the gate length of the FET's and capacitance of the $p-i-n$. The absence of a dc offset at the output of the symmetrical amplifier facilitates the cascading of multiple stages, which will further enhance the sensitivity.

\section{ACKNOWLEDGMENT}

The authors would like to acknowledge the help from Y. Ota, J. M. Geary, R. E. Frahm, and K. J. O'Brien in device packaging and in dielectric deposition.

\section{REFERENCES}

[1] R. F. Leheny et al., "Integrated InGaAs PIN-FET photoreceiver," Electron. Lett., vol. 16, p. 353, 1980 .
[2] K. Kasahara et al., "Monolithically integrated InGaAs PIN/InP MISFET photoreceiver," Electron. Lett., vol. 20, p. 314, 1984.

[3] S. Hata, M. Iketa, T. Amano, G. Motosugi, and K. Kurumada, "Planar InGaAs/InP PINFET fabricated by Be ion implantation," Electron. Lett., vol. 20, p. 947, 1984.

[4] B. Tell et al., "Monolithic integration of a planar embedded InGaAs p-i-n detector with InP depletion-mode FET's," IEEE Trans. Electron Devices, vol. ED-32, no. 11, p. 2319, Nov. 1985.

[5] C. Cheng et al., "Monolithically integrated receiver front end: InGaAs PIN amplifier," to be published in IEEE Trans. Electron Devices.

[6] Y. Inomoto et al., "A $1.2 \mathrm{~Gb} / \mathrm{s}-52.5 \mathrm{~km}$ optical fiber transmission experiment using OEIC's on GaAs on InP heterostructures," postdeadline paper presented at the Optical Fiber Communication Conf., New Orleans, LA, Jan. 1988.

[7] V. D. Mattera et al., "High speed $\mathrm{InP} / \mathrm{Ga}_{0.47} \mathrm{In}_{0.53}$ As superlattice avalanche photodiodes with very low background doping grown by continuous trichloride vapor phase epitaxy," J. Appl. Phys., vol. 60, p. $2609,1986$.

[8] J. A. Long, V. G. Riggs, and D. W. Johnston, Jr., "Growth of Fedoped semi-insulating InP by MOCVD," J. Cryst. Growth, vol. 69, p. $10,1984$.

[9] J. L. Zilko, D. L. van Haren, P. Y. Lu, N. E. Schumaker, and S. Y Leung, "The effect of gas temperature on the growth of InP by atmospheric pressure metal-organic chemical vapor deposition using trimethyl indium and $\mathrm{PH}_{3}$ sources," J. Electron Mat., vol. 14, p. 563, 1985.

[10] S. Kim et al., "A low-power, high-speed ion-implanted JFET for InPbased monolithic optoelectronic IC's," IEEE Electron Device Lett., vol. EDL-8, p. 518, Nov. 1987.

[11] S. Kim, G. Guth, and G. Vella-Coleiro, "Integrated amplifiers using fully ion implanted InP JFET's with high transconductance," IEEE Electron Device Lett., vol. 9, p. 306, June 1988. 